\title{
Hepatitis B vaccine: Coverage and factors relating to its acceptance among health care workers of a tertiary care center in North India
}

Rambha Pathak, Chintu Chaudhary, Deepak Pathania, Surendra Kumar Ahluwalia, Prabhakar K. Mishra, Arunpreet S. Kahlon

Department of Community Medicine, Maharishi

Markandeshwar Institute of Medical Sciences and Research, Mullana (Ambala), Haryana, India

Address for the Correspondence:

Dr. Rambha Pathak, Department of Community Medicine, 859, Sector-21,

Panchkula, Haryana, India. E-mail: rambha_p@yahoo.com

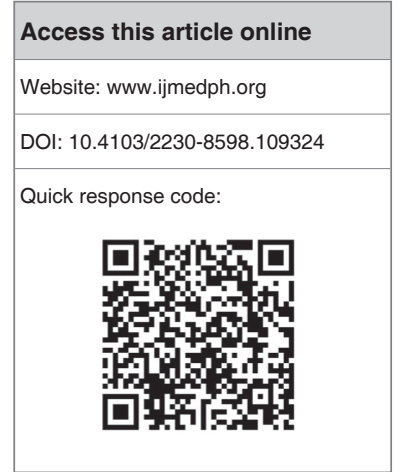

Background: Hepatitis B infection is the major cause of acute and chronic liver disease, cirrhosis, and hepatocellular carcinoma worldwide and has long been recognized as an occupational hazard among health care workers. Thus, this study was planned to evaluate the coverage of hepatitis $B$ vaccine and to study occupational, epidemiologic, and attitudinal factors in its acceptance among health care workers in a tertiary care hospital. Materials and Methods: A cross-sectional study was conducted among HCWs of MM Institute of Medical sciences and Research, Mullana. A total of 600 health care workers were selected for the study by simple random sampling method. A self designed, semi-structured interview schedule was used to collect data from the participants by interview method. Data on demographic, behavioral, and occupational exposure aspects were also collected. Results: The overall prevalence of hepatitis $B$ vaccination acceptance was $60 \%$. Only $40 \%$ of the health workers had received the full three dose vaccination schedule while $20 \%$ had received one or two doses, and $40 \%$ were unvaccinated. Protection against hepatitis B was cited the most common (54\%) reason for taking this vaccine. The most important reasons for not taking the vaccine were negligence $(41.6 \%)$, whereas the commonest $(45 \%)$ ground cited for not taking the recommended number of dosage was the lack of knowledge about the total number of doses to be taken for full protection. On multiple logistic regression, vaccine safety ( $A O R=6.57,95 \% \mathrm{Cl}=2.64-16.04)$ followed by history of exposure to blood $(A O R=5.21,95 \% \mathrm{Cl}=1.81-16.16)$ and acceptable knowledge about the disease $(A O R=4.76,95 \% \mathrm{Cl}=1.13-11.45)$ were identified as reasons for acceptance of the vaccine. Conclusions: Coverage of complete immunization was low among health care workers. Level of knowledge regarding the disease was also not satisfactory. Education programs should focus on increasing healthcare workers' perceived severity to occupational exposure to hepatitis B virus and emphasize the safety and effectiveness of the hepatitis $B$ vaccine. Vaccines should be made available free and mandatory in health organizations.

Key words: Acceptance, health care workers, hepatitis B vaccination

\section{INTRODUCTION}

Hepatitis B virus (HBV) infection is a serious global health problem. Globally, at least two billion people are estimated to have the infection, resulting in over 350 million cases of chronic infection and more than two million deaths annually. ${ }^{[1]}$ It is the tenth leading cause of death worldwide. ${ }^{[1]}$ In India, prevalence of HBsAg among the general population varies from $2 \%$ to $8 \%$, which places India in an intermediate HBV endemicity zone. India with 50 million cases is also the second largest global pool of chronic HBV infection. ${ }^{[2]}$

Since contact with body fluid of an infected person, especially infected blood, is one of the principal modes of transmission of the causative virus of hepatitis B infection, health care workers (HCWs) constitute one of the high-risk groups for this infection. ${ }^{[3]}$ High prevalence of HBV in developing countries increases the risk of occupational exposure. Thus, seroprevalence among health care workers is two to four times higher than the general population..$^{[2]}$

Towards controlling the disease, plasma-derived vaccine and later DNA-recombinant vaccines have 
been developed, and immunization has assumed a primary role as a control strategy. ${ }^{[3]}$ Realizing its necessity, the Government of India has planned to introduce hepatitis B vaccine in national immunization schedule, and pilot projects are being conducted in many parts of the country, but there are no national policies and guidelines advocating and supporting mandatory HBV immunization among health care workers, the group at highest risk of getting the infection. ${ }^{[4]}$ Acceptance of vaccination by healthcare workers is an essential issue for hospital. From India, there is very few data available on hepatitis B vaccination status of HCWs. Estimates of hepatitis $B$ vaccine coverage among health care workers are needed to calculate the proportion susceptible to HBV infection. For effective intervention, it is essential to know the factors responsible for accepting the vaccination as well as for refusal and incomplete immunization.

Thus, this study was planned to evaluate the coverage of hepatitis $B$ vaccine and to study occupational, epidemiologic, and attitudinal factors in its acceptance among health care workers in a tertiary care hospital.

\section{MATERIALS AND METHODS}

The study was conducted among health care workers - senior doctors (faculty members), junior doctors (post-graduate students, medical officers, junior residents, and interns), staff nurses, and laboratory technicians working at tertiary care hospital situated in rural part of Haryana, India. The study was conducted from June 2011 to December 2011. A cross-sectional study design was adopted. As per the data available with the medical superintendent office, there were 875 health care workers in the institute including 120 senior doctors (faculty members), 350 junior doctors (post-graduate students, medical officers, junior residents, and interns), 340 nurses including nursing interns, and $65 \mathrm{Lab}$ technicians. Proportionate numbers of participants were chosen as per the total number of staffs in different categories. Simple random sampling method was used to select study subjects in each category. Assuming a non-response rate of $20 \%, 500$ health care workers were taken up for the study, which included 86 senior doctors, 140 junior doctors, 232 staff nurses, and 42 lab technicians. A self designed, semi-structured interview schedule was used to collect data from the participants. This questionnaire was pretested in the study group and modified accordingly to elicit the desired results. For preparing the questionnaire, an extensive review of literature was done, and all the important variables were included in it. Two experts reviewed the content validity of the questionnaire before it was pretested in a sample of 35 doctors in another tertiary hospital. Cronbach's alpha was used for assessing the reliability of questionnaire.

The questionnaire included questions on age, sex, occupation, department, knowledge about hepatitis B infection, history of hepatitis infection, history of vaccination, sources of information, perceived susceptibility to infection of hepatitis $B$, and reasons for vaccine acceptance and refusal or incomplete immunization. Knowledge of hepatitis B infection refers to knowledge of health care workers about transmission of $\mathrm{HBV}$, incubation period, high-risk groups, signs and symptoms, and modes of prevention. The questionnaire included 15 multiple choice items. A score of 1 was given for a correct answer and 0 for an incorrect answer. Possible score ranged between 0 and 15 . A score of more than $65 \%$ of the maximum possible score was classified as 'acceptable knowledge' and less than $65 \%$ as unacceptable knowledge.

Questions enquiring about reasons for acceptance or refusal of vaccine and incomplete immunization were semi-structured and open-ended to get an insight into all the possible reasons and the significant variables associated with the decision. Acceptance of vaccination refers to health care workers with a history of receiving at least one dose of hepatitis $B$ vaccine. Complete vaccination refers to health care workers who had three doses of vaccine, and partial vaccination refers to those who had less than three doses of vaccine. All clinical, para-clinical, and pre-clinical disciplines were included in order to obtain a representative distribution across the hospital.

\section{RESULTS}

A total of 500 health care workers at risk for occupational blood exposures were contacted from January to March 2011. Their status of vaccination and perception regarding hepatitis $B$ vaccination was assessed. Out of the total, 440 (88\%) consented to participate in the study and successfully completed the interview

\section{Status of immunization}

Overall, the prevalence of hepatitis B vaccination acceptance was $60 \%$. Only $38.8 \%$ of the health workers had received the full three dose vaccination schedule while $21.4 \%$ had received one or two doses, and $40 \%$ were unvaccinated. Out of the total number of participants in each category, the rate of complete immunization was the highest in junior doctors (46\%), followed by nurses (44.8\%) and senior doctors (25.5\%). Only $7 \%$ of the lab technicians had received complete immunization. [Table 1]. The rate of acceptance of vaccination was higher $(75 \%)$ in females as compared to males $(62 \%)$. The HCWs working in invasive departments had twice more rate of vaccine acceptance than those working in non-invasive departments.

\section{Knowledge of hepatitis B infection}

In total, $86 \%$ of the HCW had heard about hepatitis B infection. Forty-eight percent believed that hepatitis B could be fatal. Twenty-two percent asserted that the course of disease varies from case to case while $30 \%$ had no idea about it. Responding to questions related to the mode of infection, $45 \%$ responded that it is transmitted through infected food, $32 \%$ believed it to be through contaminated water. Blood transfusion with infected blood and infected syringes can also lead to infection was told by $62 \%$ and $58 \%$ of the participants, respectively. Four percent were not sure

\begin{tabular}{lcccc}
\multicolumn{5}{l}{ Table 1: Rate of hepatitis B immunization among } \\
\multicolumn{4}{l}{ various groups of health workers } \\
\begin{tabular}{lcccc} 
Health care & Complete & Incomplete & Unvaccinated \\
workers & No. (\%) & No. (\%) & No. (\%) & \\
\hline Senior doctors & $22(25.5)$ & $52(60)$ & $12(13.9)$ & 86 \\
Junior doctors & $65(46)$ & $17(12.5)$ & $58(41)$ & 140 \\
Nurses & $104(44.8)$ & $22(9)$ & $106(45.6)$ & 232 \\
Laboratory & $3(7)$ & $16(41)$ & $23(54.7)$ & 42 \\
technicians & & & & \\
Total & $194(38.8)$ & $107(21.4)$ & $199(40)$ & 500 \\
\hline
\end{tabular}
\end{tabular}


of the mode of transmission. The disease can be prevented by using disposable syringes was known to $68 \%$ of the participants. Sixty-eight percent suggested the use of disposable syringes while $36 \%$ advocated the use of condoms as a means of preventing hepatitis B infection. Sixty percent were of the view that practicing universal work precautions is the ideal way of preventing hepatitis B infection while $72 \%$ thought of using blood from certified blood banks to prevent hepatitis B. Vaccination as a measure to prevent hepatitis B infection was espoused by $78 \%$ of the respondents. Thus, $68 \%$ of the participants had acceptable knowledge about hepatitis B infection, which included $84 \%$ of doctors, $72 \%$ of nurses, and $48 \%$ of lab technicians. As expected years of experience as health worker was also associated with high level of knowledge about the disease. Knowledge of infection was found to be significantly associated with acceptance of vaccine or completion of immunization schedule. ( $P=0.0023)$ Two percent of the workers reported to have suffered from hepatitis $B$.

The main source of information about the disease was books (82\%), followed by lectures and seminars (2\%). Only 11 participants reported to have attended special workshop on hepatitis $\mathrm{B}$ vaccine, which was organized by pharmaceutical companies for promotion of their vaccine. Fifty-four percent of the participants indicated that they would recommend vaccination to other HCWs.

\section{Perceived susceptibility}

The perceived susceptibility of the respondents regarding hepatitis $B$ virus were measured in five areas; risk of getting hepatitis $B$, potential seriousness of hepatitis B infection, frequent contact with blood and/or body fluids, needle sticks, and splash of blood and/or body fluid. The majority (68.5\%) of the participants indicated that they consider themselves at somewhat high risk of getting hepatitis $\mathrm{B}$ virus. Almost three-quarters $(73.1 \%)$ of the respondents said that they considered themselves for potential seriousness of hepatitis $\mathrm{B}$ infection as serious. There were about $21 \%$ of the participants who considered themselves as lesser susceptible for the infection. About $35 \%$ of the respondents indicated that they had more than five times per day contact with blood and/or body fluid in their jobs. The majority $(52 \%)$ of the participants indicated that they acquired more than one time needle sticks in the past, while $48 \%$ of the respondents indicated that they did not have any needle sticks, or they were not sure. Less than half (46.1\%) of the sample indicated that they had at least one time splash blood or body fluid into their mouth, eye, and nose. The author found in his study that there was a significant $(P<.002)$ relationship between the components of perceived susceptibility (risk of getting hepatitis B, potential seriousness of hepatitis B infection, splash blood and/or body fluid) and acceptance of hepatitis $B$ vaccine among healthcare workers.

\section{Reasons for accepting vaccination}

As expected, protection against hepatitis B was cited the most common $(54 \%)$ reason for taking this vaccine. Forty-two percent of the respondents received it as they believed that the vaccine available against hepatitis B is very effective. Thirty-two percent accepted the vaccine as they considered themselves at potential risk of getting the infection. Thirty percent of the participants took it as the vaccination was being made available at cheaper rate in camps or by pharmaceutical companies when administered in a group. About
$10 \%$ of the participants received vaccine as it was pre-requisite for receiving degree or appointment in medical institution, and $12 \%$ participants took the vaccine as their seniors suggested them or their friends were taking it. A few participants $(13 \%)$ took it just because it had no side-effects [Table 2]. The acceptance of vaccine among healthcare workers was found statistically significant with their age $(P=0.0122)$ and experience $(P=0.049)$. Multiple logistic regression analysis revealed that, after controlling for all other variables in the model, only three variables were significantly related to acceptance of $\mathrm{HBV}$ vaccination: History of accidental exposure to blood or blood products, acceptable knowledge about HBV infection, and perceived susceptibility to infection [Table 3].

\section{Reasons for refusal of the vaccine}

The most important reasons for not taking the vaccine were negligence $(41.6 \%)$, unavailability of vaccine through

\begin{tabular}{lcc}
$\begin{array}{l}\text { Table 2: Reasons for acceptance of hepatitis B } \\
\text { vaccine among health care workers }\end{array}$ & \\
\hline Reasons for acceptability & No. & $\%$ \\
\hline Protection against hepatitis B infection & 270 & 54 \\
High protective efficacy & 210 & 42 \\
Considered themselves at potential risk & 160 & 32 \\
Available at cheaper rates in group vaccination & 150 & 30 \\
Pre-requisite for achieving degree & 50 & 10 \\
Suggested by seniors or friends & 60 & 12 \\
No side-effects & 150 & 30 \\
\hline
\end{tabular}

\begin{tabular}{|c|c|c|c|c|}
\hline \multirow[t]{2}{*}{ Variable } & \multicolumn{2}{|c|}{ Crude } & \multicolumn{2}{|c|}{ Adjusted* } \\
\hline & OR & $95 \% \mathrm{Cl}$ & OR & $95 \% \mathrm{Cl}$ \\
\hline \multicolumn{5}{|c|}{$\begin{array}{l}\text { Accidental exposure to } \\
\text { blood or blood products }\end{array}$} \\
\hline Never & 1.00 & $1.89-14.16$ & 1.00 & $1.81-16.16$ \\
\hline Ever & 5.55 & & 5.21 & \\
\hline \multicolumn{5}{|c|}{$\begin{array}{l}\text { Knowledge about HBV } \\
\text { infection }\end{array}$} \\
\hline Unacceptable & 1.00 & $1.45-13.45$ & 1.00 & $1.13-11.45$ \\
\hline Acceptable & 4.56 & & 4.76 & \\
\hline \multicolumn{5}{|c|}{$\begin{array}{l}\text { Perceived susceptibility } \\
\text { towards hepatitis B infection }\end{array}$} \\
\hline Low & 1.00 & $1.28-6.78$ & 1.00 & $1.16-5.98$ \\
\hline High & 2.67 & & 3.67 & \\
\hline \multicolumn{5}{|c|}{ High protective efficacy } \\
\hline Yes & 1.00 & $1.14-5.33$ & 1.00 & $0.09-5.86$ \\
\hline No & 2.34 & & 2.37 & \\
\hline \multicolumn{5}{|c|}{ Economic consideration } \\
\hline No & 1.00 & $1.35-4.22$ & 1.00 & $0.98-7.22$ \\
\hline Yes & 3.44 & & 3.67 & \\
\hline \multicolumn{5}{|l|}{ No side-effects } \\
\hline Yes & 1.00 & $4.37-12.04$ & 1.00 & $2.64-16.04$ \\
\hline No & 6.25 & & 6.57 & \\
\hline
\end{tabular}


government channels (20\%), unwillingness to spend money or cost of the vaccine $(12 \%)$, low level of perceived susceptibility for getting the infection (21\%), and fear of side-effects of the vaccine $(28 \%)$ [Table 4].

\section{Reasons for incomplete immunization}

The most common (45\%) reason cited for not taking the recommended number of dosage was the lack of knowledge about the total number of doses to be taken for full protection. Twenty-eight percent of the participants could not complete immunization schedule as they did not get time while $22 \%$ forgot that they were to receive other doses too. Thirty-six percent of them were not interested in spending any money on vaccination, so they cited the reason for incomplete immunization as unavailability of vaccine through government channel [Table 3].

In multiple logistic regression, perception of vaccine safety $(\mathrm{AOR}=6.57,95 \% \mathrm{CI}=2.64-16.04)$ was identified as the most important predictor for acceptance and for willingness to recommend $\mathrm{HBV}$ vaccination to other HCWs. The odds of acceptance of $\mathrm{HBV}$ vaccination among health care workers accidentally exposed to blood or blood products was 5.21 times higher than for those not accidentally exposed. The adjusted odds of acceptance of HBV vaccination for the HCWs with 'acceptable knowledge of HBV infection' were 4.76 times higher than 'unacceptable knowledge' [Table 4].

\section{DISCUSSION}

Prevention is ultimately the most efficient and humane means toward improved health. ${ }^{[5]}$ Viral hepatitis is preventable with effective vaccines, which is available since 1982 and have proven safe to both adults and children. ${ }^{[6]}$ A significant level of non-acceptance has been reported among various groups of healthcare workers and in different settings. The results of the present study reveal that an average of $60 \%$ of health care workers had taken the three doses of the hepatitis B vaccine. Only $40 \%$ of the health workers had received the full three dose vaccination schedule while $20 \%$ had received one or two doses, and $40 \%$ were unvaccinated. The low vaccine acceptance situation has been recorded even in the groups of healthcare workers that have the greatest risk of contact with

\begin{tabular}{|c|c|c|}
\hline Reasons of refusal of the vaccine & No. & $\%$ \\
\hline Negligence & 208 & 41.6 \\
\hline Unavailability of vaccine through govt. channels & 100 & 20 \\
\hline Unwillingness to spend money & 60 & 12 \\
\hline Low level of perceived susceptibility & 105 & 21 \\
\hline Fear of side-effects & 140 & 28 \\
\hline Unsure of need of vaccine & 50 & 10 \\
\hline Believed that they had developed immunity & 60 & 12 \\
\hline Unconvinced about the efficacy & 92 & 18.4 \\
\hline Lack of knowledge about total no. of doses & 225 & 45 \\
\hline Did not get time & 140 & 28 \\
\hline Forgot & 110 & 22 \\
\hline Not interested in spending more money & 180 & 36 \\
\hline
\end{tabular}

infected body fluid such as those of the accident and emergency units, ${ }^{[7]}$ dental practitioners, ${ }^{[8]}$ and surgical residents. ${ }^{[9]}$ According to WHO estimates, it varies from $12.8 \%$ in Kenya, ${ }^{[10]} 18 \%$ in Africa, to $77 \%$ in Australia and New Zealand. ${ }^{[11]}$ In United States, $75 \%$ of HCWs at risk had received three or more doses of hepatitis B vaccine. ${ }^{[12]}$ Similarly, in Sweden, the number of HCWs who had received at least one dose is $79 \%$, but only $40 \%$ were reported to be fully vaccinated. ${ }^{[13]}$

In our study, the proportion of vaccinated HCWs differed in various occupational groups. The vaccine acceptance was the highest in junior doctors (67\%), followed by nurses $(58 \%)$ and senior doctors (49\%). Only $22 \%$ of the lab technicians had received immunization. This difference of vaccination coverage among different groups can be explained on the basis of their educational and awareness status. Age and experience of the healthcare workers also predicted acceptance of the hepatitis B vaccine. The younger healthcare workers were more likely to have been vaccinated. Resende et al. also made similar observations. ${ }^{[14]} \mathrm{HCWs}$ who had worked more years in healthcare facilities had higher rate of acceptance or completion of immunization schedule than those with lesser experience. This estimate of hepatitis B vaccination coverage in health-care workers was consistent with that obtained in the study at AIIMS; it was reported that out of $446 \mathrm{HCWs}, 224(50 \%)$ HCWs were fully vaccinated, and $28(6 \%)$ had received incomplete vaccination done. ${ }^{[15]}$ Another study at G.B. Pant Hospital, Delhi, stated that out of $2162 \mathrm{HCWs}, 1198$ (55.4\%) were vaccinated against hepatitis B. ${ }^{[16]}$ In a study conducted in Rewa (M. P.), 173 (42.4\%) out of 408 health-care workers had received partial or full course of vaccination against hepatitis $B{ }^{[17]}$

Another interesting finding in our study was difference in vaccination coverage between males and females. The rate of acceptance of vaccination was higher $(75 \%)$ in females as compared to males $(62 \%)$. This difference may be due to the fact that the overall representation of females in our study was more than males.

The current study also attempted to find out the reasons that could possibly account for poor compliance or acceptance of vaccine among doctors and nurses. Acceptable level of knowledge of hepatitis B infection was found to be significantly associated with acceptance of vaccine or completion of immunization schedule. Knowledge of $\mathrm{HBV}$ infection and $\mathrm{HBV}$ vaccination resulted in positive attitudes among the HCWs and sustained their beliefs in the safety and efficacy of vaccine. The result was consistent with the findings of Hafeez-ur-Rehman Mengal et al..$^{[18]}$ and McGrane and Staines ${ }^{[19]}$ who reported that obtaining information relating to the benefits of vaccine from an occupational health physician or from a nurse was a significant factor in acceptance of vaccine. ${ }^{[20]}$ Bradley and Kristi found that acceptance of $\mathrm{HBV}$ vaccine was strongly related to knowledge of $\mathrm{HBV}$ disease and $\mathrm{HBV}$ vaccination. ${ }^{[21]}$

This study found that the odds of acceptance of $\mathrm{HBV}$ vaccination among health care workers accidentally exposed to blood or blood products was higher than for those not accidentally exposed. One possible explanation was that they might be more conscious about HBV infection and its prevention after exposure to patient's blood. Accidental exposure might change mindset of health care workers towards acceptance of $\mathrm{HBV}$ vaccination. This study confirmed the 
findings of Hafeez-ur-Rehman Mengal et al. ${ }^{[18]}$ and Techapetpibul et al. ${ }^{[2]}$ who reported that exposure to patient blood was associated with acceptance of $\mathrm{HBV}$ vaccination by nurses.

The findings of the present study also revealed that one of the major factors for poor compliance with vaccination is the pressure of work that doctors and nurses face in the course of their daily clinical schedules. Report from the study in Croydon health district of South London ${ }^{[2]}$ supports this opinion. The finding from our study is consistent with the findings of the other authors.

\section{LIMITATIONS}

The present study is a cross-sectional study; such studies are carried out either at a single point in time or over a short period. Thus, associations identified in these studies should not be considered a causal relationship. However, there is a lack of studies that concomitantly assess the knowledge of health workers regarding hepatitis B and immunization status, as we performed in the present study. There are few reliable studies available about the hepatitis $B$ vaccine coverage among health-care workers in India. The data presented here is from north India only, so this estimate cannot be representative of the country.

\section{CONCLUSION}

Coverage of hepatitis B vaccine is low among HCWs. Lack of awareness and knowledge, attitude, and practice GAP is the reason behind this low coverage. More education about hepatitis $\mathrm{B}$ and hepatitis $\mathrm{B}$ vaccine is warranted to clear up some of the misconceptions and to promote good health. Education programs should focus on increasing healthcare workers' perceived severity to occupational exposure to hepatitis B virus and emphasize the safety and effectiveness of the hepatitis B vaccine. Enhancing accessibility may be another effective method for increasing acceptance of the hepatitis $\mathrm{B}$ vaccine. We consider that the health workers have a right to be protected against hepatitis B in view of their increased risk from occupational exposure. To this effect, national policies and guidelines advocating and supporting mandatory HBV immunization need to be put in place. Such regulations should apply to the health workers as well as to the health professionals-in training.

\section{Ethical policy}

Permission was taken from medical superintendent of the institute, and an informed and written consent was taken from the participants before conducting the study. Approval of ethical committee of the institution was sought before conducting the study. Data was collected by interview method. All analyzes were performed with the use of SPSS software, version 17.0.

\section{REFERENCES}

1. Lavanch D. Hepatitis B virus epidemiology, disease burden, treatment and current and emerging prevention and control measures. J Viral Hepat 2004;11:97-107.
2. Singhal V, Bora D, Singh S. Prevalence of Hepatitis B Virus Infection in Healthcare Workers of a Tertiary Care Centre in India and Their Vaccination Status. J Vaccines Vaccin 2011;2:118.

3. Banatvala J, Van Damme P, Oehen S. Lifelong protection against hepatitis $\mathrm{B}$ : The role of vaccine immunogenicity in immune memory. Vaccine 2000;19:877-85.

4. Sangkomkamhang US, Lumbiganon P, Laopaiboon M. Hepatitis B vaccination during pregnancy for preventing infant infection. Cochrane Database Syst Rev 2011;3:CD007879.

5. Ehreth J. The value of vaccination: A global perspective. Vaccine 2003;21:4105-17.

6. Damme PV, Herck KV. A review of the long-term protection after hepatitis A and B vaccination. Travel Med Infect Dis 2007;5:79-84.

7. Dancocks $A$, Hewitt $S$. Hepatitis $B$ immunisation status of $A$ and $E$ healthcare workers. Occup Health Lond 1994;46:20-3.

8. Echavez MI, Shaw FE, Scarlett MI, Kane MA. Hepatitis B vaccine usage among dental practitioners in the United States: An epidemiological survey. J Public Health Dent 1987;47:182-5.

9. Harward MP, Kaiser DL, Fedson DS. Acceptance of hepatitis B vaccine by medical and surgical residents. J Gen Intern Med 1988;3:150-5.

10. Suckling RM, Taegtmeyer M, Nguku PM, Al-Abri SS, Kibaru J, Chakaya JM, et al. Susceptibility of healthcare workers in Kenya to hepatitis B: New strategies for facilitating vaccination uptake. J Hosp Infect 2006;64:271-7.

11. Prüss-Üstün A, Rapiti E, Hutin Y. Estimation of the global burden of disease from sharps injuries to health-care workers. American Journal of Industrial Medicine 2005;48:482-90.

12. Mahoney FJ, Stewart K, Hu H, Coleman P, Alter MJ. Progress toward the elimination of hepatitis $B$ virus transmission among health care workers in the United States. Arch Intern Med 1997;157:2601-5.

13. Dannetun E, Tegnell A, Torner A, Giesecke J. Coverage of hepatitis B vaccination in Swedish healthcare workers. J Hosp Infect 2006;63:201-4.

14. Resende VL, Abreu MH, Paiva SM, Teixeira R, Pordeus IA. Concerns regarding hepatitis $B$ vaccination and post-vaccination test among Brazilian dentistsl. Virol J 2010;7:154.

15. Singhal V, Bora D, Singh S. Prevalence of Hepatitis B Virus Infection in Healthcare Workers of a Tertiary Care Centre in India and Their Vaccination Status. J Vaccines Vaccin 2011;2:118.

16. Sukriti, Pati NT, Sethi A, Agrawal K, Agrawal K, Kumar GT, et al. Low levels of awareness, vaccine coverage, and the need for boosters among health care workers in tertiary care hospitals in India. J Gastroenterol Hepatol 2008;23:1710-5.

17. Kumar KK, Baghal PK, Shukla CB, Jain MK. Prevalence of hepatitis B surface antigen ( $\mathrm{HBsAg})$ among Health Care Workers. Indian J Comm Med 2000;25:93-6.

18. Rehman HR, Howteerakul N, Suwannapong N, Rajatanun T. Factors relating to acceptance of Hepatitis $B$ virus vaccination by nursing students in a tertiary hospital, Pakistan. J Health Popul Nutr 2008;26:46-53.

19. McGrane J, Staines A. Nursing staff knowledge of the hepatitis $B$ virus including attitudes and acceptance of hepatitis $B$ vaccination. Development of an effective program. AAOHN J 2003;51:347-52.

20. Bradley N, Kristi J. Predictors of hepatitis B vaccine acceptance in health care workers. Med Care 1996;34:58-72.

21. Techapetpibul K, Kamolratanakul P, Ungthavorn P. Acceptance of hepatitis $B$ vaccination by nursing personnel in an area hyperendemic for hepatitis B. Chula Med J 1992;36:411-6.

22. Cimas-Hernado JE, Prieto-Gutierrez J, Rodriguez-Rodriguez JM. Acceptance of recombinant hepatitis $B$ vaccine by health personnel. Aten Primaria 1994;13:383-5.

How to cite this article: Pathak R, Chaudhary C, Pathania D, Ahluwalia SK, Mishra PK, Kahlon AS. Hepatitis B vaccine: Coverage and factors relating to its acceptance among health care workers of a tertiary care center in North India. Int J Med Public Health 2013;3:55-9.

Source of Support: Nil, Conflict of Interest: None declared. 